\title{
Determining Appropriate Parameters to Elicit Linear and Circular Apparent Motion Using Vibrotactile Cues
}

\author{
Masataka Niwa $^{1} \quad$ Robert W. Lindeman ${ }^{2} \quad$ Yuichi Itoh $^{1} \quad$ Fumio Kishino $^{1}$ \\ ${ }^{1}$ Graduate School of Information Science and Technology, Osaka University \\ ${ }^{2}$ Department of Computer Science, Worcester Polytechnic Institute
}

\begin{abstract}
This paper reports on two experiments we conducted to look at how to design effective linear and circular apparent-motion displays. Using a two-tactor array on the upper arm, the first study found that a time interval of greater than $400 \mathrm{~ms}$ before repeating the directional sequence is required for subjects to achieve $95 \%$ proficiency of direction identification over a range of Duration of Stimulus (DOS) and Stimulus Onset Asynchrony (SOA) values. The second study looked at the number of tactors necessary in a circular tactor array on the upper arm for people to correctly identify the direction of the rotation. We found that subjects could achieve a proficiency approaching $100 \%$ across a large range of DOS and SOA values with four tactors, using a circuit-completion time of $400 \mathrm{~ms}$ or longer. These findings can be used by interface designers to realize information displays that either stand on their own, or work in combination with visual and/or audio displays.
\end{abstract}

KEYWORDS: Apparent motion, phantom sensation, vibrotactile, DOS, SOA.

INDEX TERMS: H.5.2 [User Interfaces]: Haptic I/O; H.1.2

[User/Machine Systems]: Human factors; I.3.6 [Methodology and Techniques]: Interaction techniques.

\section{INTRODUCTION}

One can provide additional information to visual and audio displays using vibrotactile stimulation. Alternatively, vibrotactile stimulation can be used in situations where visual or audio displays are inappropriate. The use of vibrotactile stimulation is especially useful in conditions where the user is attending to other tasks or displays, as it stimulates the skin, which has a much larger stimulation field than the eyes or ears. Finally, vibrotactile stimulation is useful in situations where information should be displayed in a private manner, such as in public spaces.

With regard to vibrotactile displays, much research has been done in the area of sensory substitution, but often these devices are large in size, such as TVSS [1]. More recently, many consumer devices, such as mobile phones and video-game controllers, incorporate simple DC-motor-type vibration units (tactors) that are readily available, inexpensive to purchase, and relatively easy to control using straightforward circuitry. Because of these factors, there has been a resurgence of research into the use of vibrotactile stimulation. For example, Rupert [2] used a vest with tactors sewn into it to relay gravity information to pilots performing aerial maneuvers. Piateski \& Jones [3] used tactor

\footnotetext{
${ }^{1}$ e-mail: \{niwa.masataka, itoh, kishino\}@ist.osaka-u.ac.jp

e-mail: gogo@wpi.edu
}

arrays on the arm and torso to aid users in navigation tasks. Brown et al. [4] used rhythmic patterns and changes in amplitude to explore the use of multiple vibration attributes to relay information to the fingertips.

Our own previous work [5] suggests that information displays using apparent motion could be a feasible approach for presenting multiple types of information through the manipulation of various apparent-motion parameters. Apparent motion is a phenomenon that a stimulus appears to move smoothly, even though the individual display units are discrete. Though there is space between each unit, by employing appropriate time intervals, the stimulus appears to be continuous. Examples of apparent motion in the visual domain are quite common. For example, old-style movie marquees used strings of lights that would blink using such a pattern that the light seemed to be moving along the length of the string. Road-construction sites, or curves on the highway, often use this same technique. Sherrick $[6,7]$ and Kirman [8] performed studies of apparent motion using vibrotactile stimulation on the skin, and showed that this phenomenon can elicit feelings of continuous motion.

In [9], Sherrick also reports that the feeling of apparent motion is controlled by two factors (Figure 1). The Duration of Stimulus (DOS) is the amount of time an individual tactor is vibrating. Stimulus-Onset Asynchrony (SOA) is the time difference between one tactor turning on and the next tactor in the sequence turning on. Using three rings of tactors mounted on the arm, we previously measured the ability of users to identify (1) the speed of apparent motion, and (2) individual patterns of apparent motion, including clock-wise/counter-clock-wise movement, and up/down movement from ring to ring [5].

Based on this research, the current study looks at determining the proper timing values (DOA, SOA, etc.) to produce linear apparent motion. In addition, we attempt to determine the sufficient number of tactors necessary to elicit circular apparent motion around the arm.

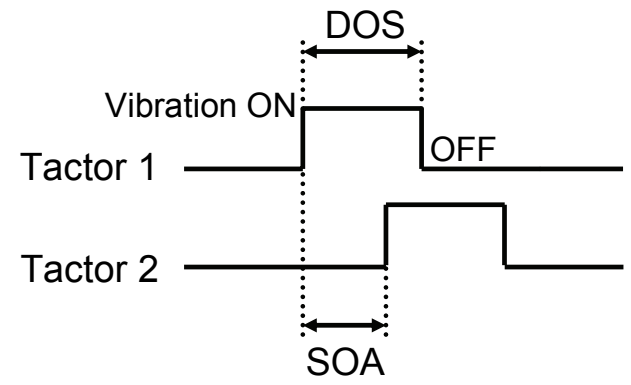

Figure 1: Duration of stimulus (DOS) and stimulus onset asynchrony (SOA). 


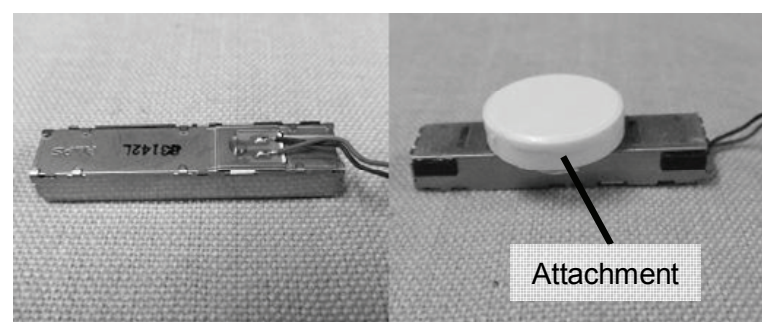

Figure 2: Tactor and attachment.

\section{TACTORS USED IN OUR WORK}

The current study uses the Force Reactor tactors (ALPS AF-Series, Type L) shown in Figure 2. These are the tactors found in the Rumble Pak of the Nintendo DS handheld game system. The technology is similar to voice-coil-type tactors, and provides a faster response time than DC-motor-type tactors. They provide a solid platform for studies of apparent motion where very finegrained control of DOS and SOA is required. The vibration frequency of the Force Reactor tactors is in the range of 200$300 \mathrm{~Hz}$, which is the range of the underlying mechanoreceptors in the skin that sense vibration of this kind $[10,11]$. In this experiment, we will use $300 \mathrm{~Hz}$ as the vibration frequency.

As Figure 2 shows, this tactor has a long, thin form factor, which would produce a stimulus field on the skin that is long in one direction, and narrow in another. Therefore, we mount each tactor onto a rigid, ceramic disk ( $17 \mathrm{~mm}$ diameter $\times 4 \mathrm{~mm}$ height), similar in dimension to previous work [12], which used a voicecoil tactor (NEC/Tokin MMA-33). Using the disk makes a smaller, symmetric stimulus field.

\section{EXPERIMENT 1: ContinUOUS, LINEAR Motion DisPlay}

\subsection{Objectives of Experiment 1}

When multiple tactors are used in a linear array, and the tactors are triggered in order, apparent motion can be displayed. However, if the sequence is continuously repeated, and the time interval between successive sequences is too short, the stimulus can be felt to be moving back and forth (Figure 3). Therefore, this experiment attempts to identify the minimum time interval for users to perceive the motion as being linear in a single direction, as opposed to back and forth.

\subsection{Method of Experimentation}

As shown in Figure 4, the tactors were affixed on the upper arm using double-sided tape. A single tactor was placed on the front of

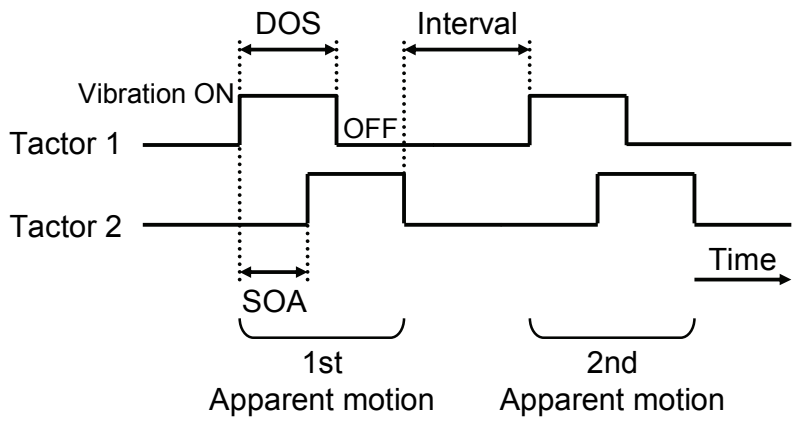

Figure 3: Time interval between sequences. the bicep 50mm from the "elbow crease." In addition, a second tactor was mounted $150 \mathrm{~mm}$ from the elbow crease in line with the first tactor. Subjects were seated with their hands in their laps, and wore headphones playing white noise to block out any audio cues.

The stimuli were delivered to allow subjects to sense continuous apparent motion in one direction. Two stimulus sequences were used. Referring to Figure 4, movement in a DOWN direction was delivered using the sequence of Tactor A followed by Tactor B. For an UP motion, the sequence was Tactor $\mathrm{B}$ followed by Tactor A. To reduce the possibility that subjects would use the first tactor in the sequence to determine the direction, the tactor in a given sequence that was triggered first was varied. For example, even if the DOWN sequence was displayed, sometimes the sequence would begin with Tactor B. Because the sequence was repeated until the subject declared the direction, this approach seems valid.

Four pairs of DOS/SOA combinations (i.e., movement speed of the stimulus point across the tactors) were used: 100/100, 200/200, $400 / 300$, and $800 / 400 \mathrm{msec}$. The DOS values have been shown to be good candidates for eliciting apparent motion [12]. In addition, the time interval between sequences varied over 11 levels: 0,100 , $200, \ldots, 1000 \mathrm{msec}$.

Subjects were given a choice as to whether they felt the stimulus point was moving UP or DOWN. Ten subjects (nine male, one female) in age from 20-29 took part in the experiment. In summary, each subject experienced four DOS/SOA combinations, two directions, two different starting points, and eleven time intervals, giving a total of 176 combinations. Each subject received each combination four times, giving a total of 704 data points per subject. The combinations were displayed in random order.

\subsection{Results and Analysis of Experiment 1}

The results are shown in Figure 5. The horizontal axis shows the time interval, and the vertical axis is the percentage of correct answers, calculated from all the responses from all the subjects. Each plot shows a different DOS/SOA combination. As shown in the figure, among all DOS/SOA combinations, when the time interval is $0 \mathrm{msec}$, the percentage of correct answers is lowest. As the time interval increases, the percentage of correct answers also

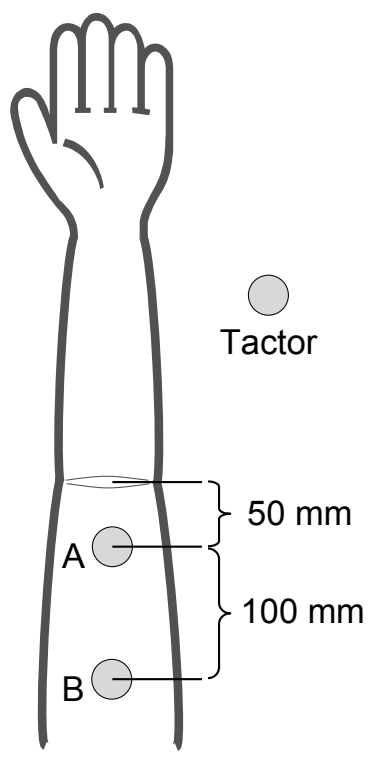

Figure 4: Tactor placement for Experiment 1. 


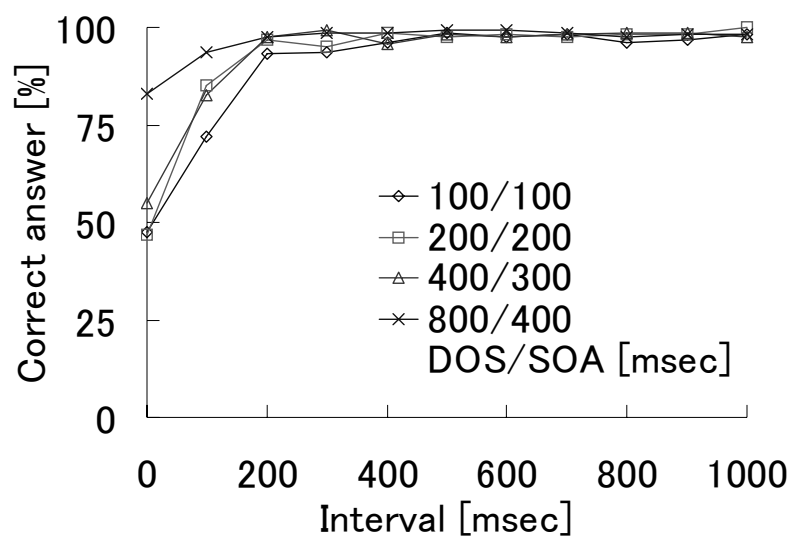

Figure 5: Correct-answer percentage of all subjects by stimulus.

increases. Furthermore, when the DOS/SOA are $800 / 400 \mathrm{msec}$, a percentage of greater than $90 \%$ was achieved for time intervals greater than $100 \mathrm{msec}$. In the other three DOS/SOA combinations, greater than $90 \%$ was achieved for time intervals greater than $200 \mathrm{msec}$. A correct-answer percentage of greater than $95 \%$ was achieved for time intervals greater than $400 \mathrm{msec}$, regardless of DOS/SOA levels.

We also found very strong evidence that the starting point for the sequence has a dramatic influence on recognition rates. For example, in the DOWN cases, when the starting point was Tactor A, recognition rates for increasing DOS/SOA combinations were $73 \%, 73 \%, 88 \%$, and $96 \%$ when the time interval was $0 \mathrm{msec}$. When the time interval was $100 \mathrm{msec}$, these rates jumped to $95 \%$, $95 \%, 98 \%$, and $99 \%$, respectively. Comparing these values to the overall numbers shown in Figure 5, we can confirm that subjects used the sequence starting point to help determine the direction of the stimulus movement.

In summary, when one provides continuous, linear stimulus in a single direction, people should be able to correctly identify the apparent motion direction more than $95 \%$ of the time with a time interval greater than $400 \mathrm{msec}$. When subjects correctly sense the initial stimulus point of the sequence, they can achieve similar results with even shorter time intervals.

\section{EXPERIMENT 2: NUMBER OF TACTORS REQUIRED FOR CIRCULAR MOTION DISPLAY}

\subsection{Objectives of Experiment 2}

In order to display circular apparent motion around the upper arm, tactors can be deployed in a ring around the arm. However, space on the upper-arm is limited, and placing many tactors on the upper arm makes the system more cumbersome. Ideally, we would like to use as few tactors as possible, while still retaining high correctidentification rates. Therefore, this experiment aims to determine the appropriate number of tactors needed to support effective circular apparent motion perception.

\subsection{Method of Experimentation}

As shown in Figure 6, a ring of tactors was placed around the bicep $150 \mathrm{~mm}$ from the elbow crease. The ring consisted of 3, 4, or 5 equally spaced tactors. Subjects were seated with their hands in their laps, and wore headphones playing white noise to block out any audio cues.

Each sequence was repeated five times (i.e., five loops around the ring) for each trial. The speed of the stimulus movement varied based on two factors: SOA and the number of tactors. Eight

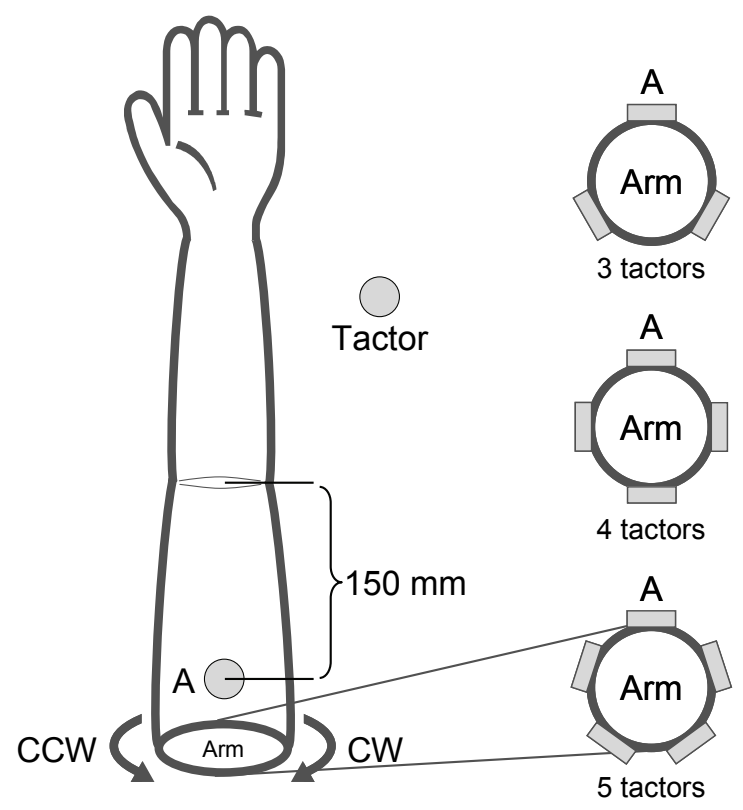

Figure 6: Tactor placement for Experiment 2.

Table 1: Combinations of DOS and SOA for Experiment 2. (Circuit-Completion Time $=$ SOA $\times$ Number of Tactors $)$

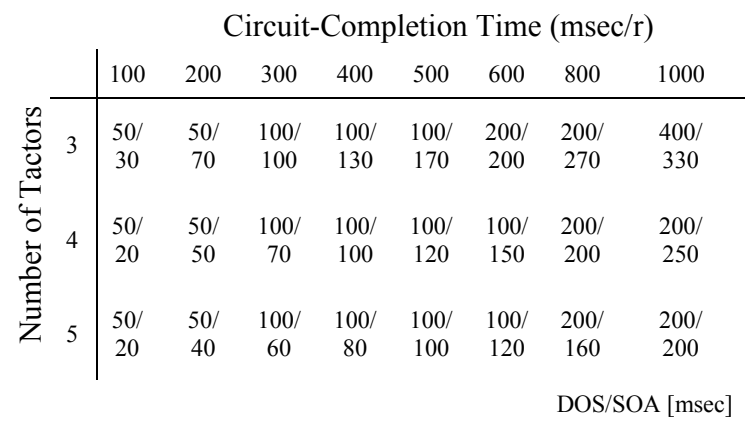

different circuit-completion times were used. In order to properly compare results from different numbers of tactors, the time for one circuit around the ring can be computed as SOA * number of tactors. As shown in Table 1, the stimulus was provided by combining DOS and SOA. If the time of one circuit was not evenly divisible by the number of tactors, the SOA was adjusted to get as close as possible to the target circuit-completion time.

Subjects had to answer with either CLOCKWISE or COUNTER-CLOCKWISE following each trial. Ten male subjects in age from 20-29 took part in the experiment. In summary, each subject experienced eight different circuitcompletion times and two directions, with 5 repetitions, giving 80 combinations, which were displayed in random order. Also, each subject performed the experiment with a 3-, 4-, and 5-tactor rings.

\subsection{Results and Analysis of Experiment 2}

The results are shown in Figure 7. The horizontal axis shows the circuit-completion time, and the vertical axis shows the percentage of correct answers. Each plot shows the number of tactors in the ring. As the figure shows, using the fastest circuitcompletion time $(100 \mathrm{msec} / \mathrm{r})$, regardless of the number of tactors, the percentage of correct answers is about $50 \%$, meaning the 


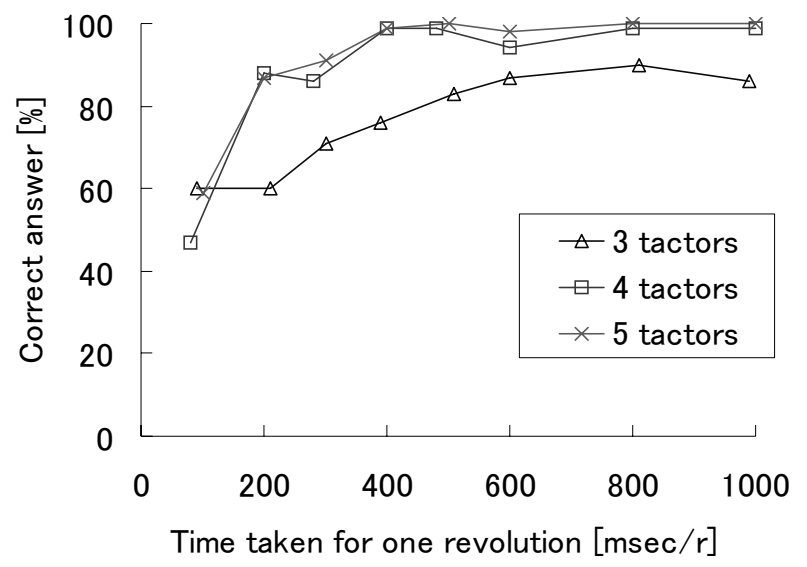

Figure 7: Correct-answer percentage of all subjects by number of tactors.

answers were basically random guessing. Based on these numbers, we can conclude that this speed is below the threshold of what humans can use for direction recognition.

When the number of tactors is three, the correct-answer percentage is lower than when the number of tactors is four. Furthermore, when the number of tactors is greater than four, if the circuit-completion time is $400 \mathrm{msec}$ or longer, subjects achieved close to a $100 \%$ correct-answer percentage. In contrast, when the number of tactors is three, the best correct-answer percentage was $90 \%$ across all circuit-completion times. Therefore, in order to display circular motion correctly, at least four tactors are necessary, and the time to complete one circuit needs to be $400 \mathrm{msec}$ or longer.

Almost the same results were obtained using five tactors versus four. Some subjects commented after the experiment that when comparing three tactors versus four, it was easier to determine direction when there were four, but between four and five, there was not much difference. Based on these findings, in order to display circular motion on the upper arm, four tactors are sufficient, and the addition of one more tactor is probably not necessary.

\section{Conclusion}

These studies built on results obtained by ourselves and others on determining appropriate DOS and SOA values for vibrotactile stimuli. We determined the necessary time interval between stimulus sequences in order to correctly identify continuous linear apparent motion on the upper arm. The results show that a 400 msec or longer time interval leads to $95 \%$ or better correct direction identification.

Next we attempted to determine the minimum sufficient number of tactors to correctly identify the direction of circular apparent motion on the upper arm. The results show that four or more tactors should be used, and the circuit-completion time should be $400 \mathrm{msec}$ or longer.

These findings can be used by interface designers to realize information displays that either stand on their own, or work in combination with visual and/or audio displays. In addition, these findings can be used to deliver motion-following or navigation information for applications such as sports training [13]. In the future, we would like to investigate whether the same results are obtained from tactors placed on other parts of the body.

\section{REFERENCES}

[1] P. Bach-y-Rita, C.C. Collins, F.A. Saunders, B.W. White, L. Scadden. Vision substitution by tactile image projection. Nature 221, pages 963-964, 1969.

[2] A.H. Rupert. An instrumentation solution for reducing spatial disorientation mishaps. IEEE Engineering in Medicine and Biology 2000, pages 71-80, 2000.

[3] E. Piateski, L. Jones. Vibrotactile pattern recognition on the arm and torso. Proceedings of the First Joint Eurohaptics Conference and Symposium on Haptic Interfaces for Virtual Environment and Teleoperator Systems, pages 90-95, 2005.

[4] L.M. Brown, S.A. Brewster, H.C. Purchase. A first investigation into the effectiveness of tactons. Proceedings of the First Joint Eurohaptics Conference and Symposium on Haptic Interfaces for Virtual Environment and Teleoperator Systems, pages 167-176, 2005.

[5] L. Kohli, M. Niwa, H. Noma, K. Susami, K. Hosaka, Y. Yanagida, R.W. Lindeman, Y. Kume. Towards effective information display using vibrotactile apparent motion. Proceedings of the 14th Symposium on Haptic Interfaces for Virtual Environment and Teleoperator Systems, pages 445-451, 2006.

[6] C.E. Sherrick, R. Rogers. Apparent haptic movement. Perception \& Psychophysics, Vol. 1, pages 175-180, 1966.

[7] C.E. Sherrick. Bilateral apparent haptic movement. Perception \& Psychophysics, Vol. 4, pages 159-160, 1968.

[8] J.H. Kirman. Tactile apparent movement: The effects of interstimulus onset interval and stimulus duration. Perception \& Psychophysics, Vol. 15, No. 1, pages 1-6, 1974.

[9] C.E. Sherrick. Studies of apparent tactual movement. In D.R. Kenshalo (Ed.), The Skin Senses, pages 331-344. Springfield, IL: C.C. Thomas, 1968.

[10] C.E. Sherrick. Variables affecting sensitivity of the human skin to mechanical vibration. Journal of Experimental Psychology, 45, pages 273-282, 1953.

[11] G.D. Goff. Differential discrimination of frequency of cutaneous mechanical vibration. Journal of Experimental Psychology, 74, pages 294-299, 1976.

[12] M. Niwa, Y. Yanagida, H. Noma, K. Hosaka, Y. Kume. Vibrotactile apparent movement by DC motors and voice-coil tactors. Proceedings of ICAT 2004, pages 126-131, 2004.

[13] J. Kume, M. Konuki. Sports training using vibrotactile stimulation. Proceedings of the 10th Annual VRSJ Meeting, (in Japanese), pages 50-54, 2005 